\title{
The Analysis of Modal Adverb "heku/hebi" and Its Application in Teaching Chinese as a Foreign Language
}

\author{
Song Jie, Xiao Jiugen* \\ Research Center of Language and Language Life, Jiangxi Normal University, Nanchang, China \\ Email address: \\ 41094067@qq.com (Song Jie), jxsdxjg666666@sina.com (Xiao Jiugen) \\ ${ }^{*}$ Corresponding author
}

To cite this article:

Song Jie, Xiao Jiugen. The Analysis of Modal Adverb "heku/hebi" and Its Application in Teaching Chinese as a Foreign Language. Science Innovation. Vol. 8, No. 4, 2020, pp. 102-108. doi: 10.11648/j.si.20200804.14

Received: June 19, 2020; Accepted: July 31, 2020; Published: August 10, 2020

\begin{abstract}
As a special kind of modal adverbs in Chinese, rhetorical adverbs have been used since the pre-qin dynasty. They are frequently used in spoken and written languages, and have their unique features in both form and usage. The adverb "heku/hebi" has been studied by predecessors and has been very enlightening. However, it is a pity that the adverb has not been put on the three planes of semantics, grammar and pragmatics to analyze the similarities and differences between the adverb and the adverb, nor has it been combined with the theoretical discussion and practical application of teaching Chinese as a foreign language. Therefore, it is necessary to carry out the research in this respect, not only has the theoretical significance, but also has the application value. The adverb "heku/hebi" has both commonness and individuality in its semantic, grammatical and pragmatic aspects. By analyzing and comparing their general commonalities and unique characteristics, and applying them to teaching Chinese as a foreign language, students can not only grasp their semantic features, grammatical features and pragmatic features, but also greatly improve their practical application ability.
\end{abstract}

Keywords: Rhetorical Tone Adverb, "heku/hebi”, Analysis, Teaching Chinese as a Foreign Language, Using

\section{浅析“何苦”与“何必”及其在对外汉语教学中的应用}

宋洁, 肖九根 ${ }^{*}$

江西师范大学语言与语言生活中心, 南昌, 中国

邮箱

641094067@qq.com（宋洁）,jxsdxjg666666@sina.com（肖九根）

摘要: 汉语中的反诘语气副词是语气副词中较为特殊的一类, 从先秦沿用至今, 在口语和书面语中使用频率极高, 无 论形式还是用法都有其独具的特点。反诘语气副词“何必/何苦”, 前人时贤都作过一些研究, 也给人以很大启迪, 但遗 憾的是未将其置于语义、语法、语用三个平面上去剖析其间的异同，也未将其同对外汉语教学的理论探讨与实际运用 结合起来。因此，对之进行这方面的研究很有必要，这不仅有理论意义，还有应用价值。反诘语气副词“何必/何苦”在 其语义、语法、语用方面既有共性, 也有个性。通过分析比较二者的一般共性与独特个性, 并将其运用于对外汉语教 学实践中, 不但有利于学生把握其语义特征、语法特征和语用特征, 而且还能极大地提高他们的实际运用能力。

关键词：反诘语气副词, “何必/何苦”, 分析, 对外汉语教学, 运用 


\section{1. 引言}

近些年来, 随着汉语副词研究的不断深入, 语气副词 也引起了学者们的关注, 这在他们出版的专著和虚词词典 中都占有一定的份量。概而观之, 语气副词的研究主要表 现在以下四个方面:

一是对语气副词进行定性分析。例如, 王力（2011） 指出, 一般副词“表示一种事实义, 又是完全不带情绪的”, 而像“可、难道”一类词是“完全缺乏实义的, 带有情绪的”。 他把后一类词称为“语气副词”。[1]（p9）史金生(2003)根 据语义和说话人对说话内容的某种情感或态度, 从表达功 能的角度对语气副词进行了逐层分类，他把“难道、岂、 何必”之类归入疑问语气副词。[2]

二是对语气副词进行多维度分析。例如, 肖奚强(2003) 从模态的角度出发, 将语气副词称之为模态副词, 如把“难 道、岂、何必”等称之为疑问模态副词。文章中, 作者对 其在语义表达、句法搭配、语用功能等方面都作了一些分 析。不过, 论述略显简单, 作者未对这三个系列词之间的 联系与区别作详尽的阐述。[3]何雪飞（2019）对早期现代 汉语副词的使用情况作了一般性的考察, 并从单、复句的 副词句位中总结其特征及成因, 不过论文阐释某些现象有 待进一步深入。[4]

三是对语气副词进行单个分析。例如, 范宁（2010） 分析了“何X”如何由两个独立的句法成分逐渐演变成一个 词的词汇化过程。作者认为, 除部分尚未完成还在词汇化 完成过程中之外, “何X”大部分已经完成了词汇化过程,

（如“何以”）。不过，作者尚未对该类词进行必要的共时 分析。[5](p5)张晓东（2019）以《现代汉语八百词》为基 础, 从语义和句法两方面分析对外汉语教学中的语气副词 “倒”, 并提出了教学方法, 当然遗憾的是作者未从语用方 面去作分析。[6]武远佳 (2019) 从历时的角度考证了语气 副词“早晚”出现的时代及其形成过程和重要机制。[7]

四是对语气副词的比较分析。例如, 袁静 (2018) 对 语气副词“不必”“无须”从句法、语义和使用频率方面作了 共时比较分析, 但未涉及语用预设方面的讨论。[8]朱宇贺 （2019）从认知视角对语气副词“想来”“看来”的词性变化 和语义特征作了比较分析, 稍嫌不足的是缺乏句法、语用 方面的分析。[9]

有关语气副词“何”的分析大多散见于前辈先贤的专 著中, 多是历时方面的研究, 还有学者对现代汉语中的“何 $\mathrm{X}$ ”作了分析研究, 但主要着眼分析具有共同语义“何 X”中 的某个小类, 而没有从整体上对“何X”作系统分析, 也很 少有人对其形成中的共性与个性差异作具体考察。

通过对前人研究的考察可以发现, 前人研究的不足之 处主要表现在: 一是缺乏类似的反诘语气副词之间的比较, 忽略了它们之间的细小差异; 二是研究现代的论文较多, 多数是从一个角度入手, 缺乏历时分析; 三是没有提供在 对外汉语教学中如何教授这类词的切实可行的教学方法。

本文从三个平面揭示“何苦/何必”这组词所具有的共 性和差异, 其研究意义和价值主要有二: (1) 对其研究 可以运用于对外汉语教学之中, 通过对比分析其异同, 更 加明确其意义和用法, 也使汉语学习者在深入理解“何”类 反诘语气副词的基础上正确地加以运用。（2）为类似的
反诘语气副词研究提供一定的参考, 进一步挖掘、丰富语 气副词的分析研究。

\section{2. 语气副词“何必/何苦”的三个平面分析}

对“何必”与“何苦”进行研究, 涉及其句法特征、词语 搭配、语义特征以及语用预设等方面。

本文语料来自北京大学现代汉语及古代汉语语料 库、北京语言大学 $\mathrm{BCC}$ 语料库及北京语言大学 HSK 动 态作文语料库。

\section{1. 语气副词“何必/何苦”的句法特征分析}

\subsection{1. 语气副词“何必/何苦”句法功能}

“何必”位于句首, 后接动词短语或形容词短语, 强调 了说话者的态度, 通常认为没有必要做某事。

（1）皮日新道: “我有钱, 哪里也好捧角, 何必一定 要捧她。”

“何必”位于句首时, 还可以作句首状语, 其后直接接 主语。

（2）让他们把水弄到楼上来好了, 何必你又上楼下 楼。

“何必”位于句中是常态, 常出现在主语后谓语前, 如 例（3）; 还可以出现在谓语的位置上, 如例 (4) :

(3) 理查说: “这我可不知道, 那是你们所举行的丧 礼, 我何必关心呢?

（4）逸芳回答: “不过，她何必呢！对于她又没有好 处。”

书面语中，很少见到“何必”位于句末的情况，“何必” 通常位于句末是因为省略了谓语, “何必”位于句末在口语 中更为常见, 表示说话人对受话人的劝告, 如例 (5):

（5）她抓住小蹊的手说道: “小妹你才三十岁，何 必......”

“何苦”同“何必”一样能放在句子开头，“何苦”位于句 首是一种常态, 在语料库的例子中抽取 200 条, 有 182 条是 “何苦”置于句首的，如例（6）：

（6）人生而自由，何苦不及时行乐?

“何苦”位于句首, 后接短语, 不过前面通常须有主语, 如例 (7):

（7）黎蒂又接着说: “罗菩! 你何苦也学别人那样俊 呢?"

“何苦”位于句中的情况并不多,一般位于主语后谓语 前, 如例 (8) :

（8）娄知县何苦命令手下杀人呢?

还有一种情况是“何苦”处在谓语的位置, 一般处在 “这（又）/那（又）十何苦”的结构之中, 如例:

（9）老蒋早已决定了, 你若反驳, 这又何苦呢!

（10）夫人笑说: “那又何苦。”

“何苦”位于句末在书面语中较少见，一般位于句末的 话是表示一种劝慰、遗憾, 意犹未尽的语气,如例 (11):

（11）我也可以选择背信弃义, 可我做不到啊! 都是 一家人，何苦...... 


\subsection{2. 语气副词“何必/何苦”独用及其与语气词连用}

“何必”与语气词“呢”连用很常见, 通常是时断时续的 连用, 如例 (12) :

（12）现已是雨季，少有太阳，何必还晒被子呢?

虽然副词一般做状语修饰谓语不单独使用, 但是作为 表示语气的副词, “何必”是可以单用的。“何必”单独使用 时, 通常也要加上语气词, 如例:

（13）每天你都白天睡觉, 半夜起来看书, 何必呢?

例（13）的“何必”加上语气词“呢”可独立成句, 并且 加强了反诘的语气。

“何苦”单独使用时, 一般要和语气词连用, 加强表示 劝慰、无奈、规劝的语气，如例:

（14）“何苦！假如刚才你就让它走的话.....”

“何苦”除了经常与“呢”连用外，也和“来着”“来哉”等 语气词连用, 不过使用频率不高, 通常是在口语中使用, 如例:

（15）想到弟弟还在替他养家，这是何苦来着?

（16）有大路不走非要走小路, 何苦来哉?

\subsection{3. 语气副词“何必/何苦”与其它副词连用}

“何必”多与“又”连用，大都置于“又”之后。当“又”位 于“何必”之前时表达说话者的无奈。

在2973例“何必”语料中，“又”位于“何必”前的有 574 例, 如例:

（17）凤举叹道: “你又何必伤心？连老七他自己, 还看得十分平淡呢。”

例（17）“何必伤心”只能单纯地表达说话者觉得做某 事没有必要的意思, 加上“又”则加强了其表义作用, 如果 去掉“又”语势就减弱了。[10](p28)

（18）你要杀要别都行, 又何苦羞辱我们?

例（18）加了“又”之后, 也就强调了规劝的语气。

\section{2. 语气副词“何必/何苦”的语义特征分析}

《现代汉语虚词词典》对“何必”的解释是：“为什么 一定要”, 用于反问语气表示不必要, 相当于“不必”错误!未找 到引用源。 。如例:

（20）小曼说：“我不相信在上海找不到一件礼服。 何必一定要和我悉心挑选的衣服一样呢? ”

(21) 安尼心想: 总会有变通之法的, 何必自寻烦恼?

从上述例句可以看出, “何必”表示做某件事没必要, 例（20）言外之意是“没必要穿与我一样的礼服”; 例 (21) 意即“只要找办法总会有的, 没必要自寻烦恼”。不仅如此, “何必”还可以表示做某事不值得。如例:

（22）“童家霆, 别那么多情!何必再去想她呢? ”

（23）自己的委屈最好是放在心中, 何必让家人也一 起难过呢。

例（22）意即“她已有所爱的人了，你不值得去想一 个不爱自己的人”; 例 (23) 表示的是“有委屈要自己忍着, 让家人一起难过不值得”。这些例句均表达一种劝慰、告 诫的语义。也就是说，上述“何必”的例句均带有一种主观 劝告、建议或强调的语义特征。
对于“何苦”一词，《现代汉语虚词词典》释之为: 用 于反问句，表示没必要自讨苦吃。如例:

（24）她只是虚无缥组之物, 何苦为这样的女人再伤 情呢?

“何苦”语义上表示说话人主观上认为做某事会让人 自找苦吃, 带有劝解之意。如 (24) 例说话人认为所爱慕 的人虚无缥纱, 没必要为她而伤心。“何苦”所传达的语义 也带有一定的主观性, 具有委婉规劝的语义特征。

\section{3. 语气副词“何必/何苦”的语用特征分析}

言语中的任何词语都离不开一定的语用环境的, 即使 虚词也不例外。[11]例如:

（25）紫玉称她为老大姐，她不满的说：你叫我大姐 本来就不对，你何必又加个“老”字?

此话的语意蕴含是：尊我为“姐”得体, 可称谓前来个 “大”就变味了, 让人有老之将至的感觉; 而这还不够, 居 然还以“老”相称，这不是明捧实损吗？故而“不满”。

(26) 他说瑶瑶, 你还是上学的年纪, 应该认真读书, 何必去竞选"上海小姐"?

此话的语义显而易见：还在上学的“瑶瑶”有着强烈意 愿要去竞选上海小姐, 不过说话者从骨子里反对, 认为她 去竞选不是时候。如“他”的话起了作用, 则会阻止她行动 的实现, 否则相反。从上述句子可知, “何必”预设的语义 特征一般有: 动作已完成或未完成; 说话人认为动作没必 要实现; 受话人认为动作可以实现, 并且在他看来这是合 理的。

众所周知, 反诘语气副词主要表达某种语气、态度, 并非一种具体的语义, 所以语义预设的影响比语用预设的 要小, 可以通过增删语气词来对比两者之间的差异。例如:

（27）黎蒂说: “你何苦也学别人那样俊呢?”

黎蒂说: “你怎么也学别人那样俊呢?”

例（27）如删去“何苦”，全句只有反问语气; 一旦加 上不仅表达反问语气, 而且还多了劝慰、惋惜的语气。

\section{3. 语气副词“何必/何苦”的对比分析}

\section{1. 语气副词“何必/何苦”的相互替换}

多数情况下，“何苦”可以替换“何必”，表没有必要做 某事。例如:

（28）青青气道: “要不是有急事, 一小时打七八次 电话给你?"

青青气道: “要不是有急事, 我一小时打七八次电话 给你?"

从“何必”和“何苦”的语义特征看，虽然二者都表达没 必要做某事之意。但是，“何必”倾向于没必要做某事，而 “何苦”则更倾向于不值得做某事，通常是在做了某事会带 来何种消极后果的情况下使用的。所以，有时候“何必”不 能替换“何苦”。[12]例如:

（29）“真那样的话，何必不直接问问?”

“真那样的话，何苦*不直接问问? ”

（30）“何必呢? 只是死了个仆人！真是大题小做！” 烈格雷说。 
“何苦呢*? 只是死了个仆人! 真是大题小做！”烈格 雷说。

“何苦”表达做某事不值得，所以例（29）表示“为什 么不直接问问”的“何必”不能用“何苦”替换, 且其语气也没 有“何苦”那么重。所以，例（30）的“何必”也不能为“何苦” 所替换。又如:

（31）老汉说: “婶子, 你何苦用头去撞地, 你车上 过保险, 走正常程序, 怕啥呢! ”

老汉说: “婶子, 你何必*用头去撞地, 你车上过保险, 走正常程序, 怕啥呢! ”

例（31）是对已发生的事表示惋惜, 即以头去撞地的 动作已经发生了, 不能被表达对可能还未发生的事以示劝 说的“何必”代替。[13]

\section{2. 语气副词“何必/何苦”的语义对比分析}

通过上文对“何必”与“何苦”的语义分析，可以将其语 义特征列表于下:

表1 “何必”与“何苦”的语义特征。

\begin{tabular}{lllll}
\hline 何必 & 主观性 & 规劝 & 强调 & 建议 \\
何苦 & 主观性 & 规劝 & 惋惜 & 无奈 \\
\hline
\end{tabular}

3.2.1. 何必

“何必”与“何苦”最大的不同在于其表达的语气, 此外, 两者在句子中出现所依据的条件也不同。

那么, “何必”表达的语气以及出现的语义条件是什么 呢？请看下面例子：

（32）你何必歳眉?收起你的哀怨。

例（32）省略了事情发生的前提, 说话人认为没有必 要歳眉, 究竟为什么不要歳眉, 语境中没有提到, 但也不 需去深究, 因为“何必”已将语义完整地表达出来了。又如:

（33）“我一个糟老头去哪住都一样，何必为我吵架 伤感情。”

从这个例子可以看出, 句子的前提是“我一个老头在 哪住都没差别”, “为老头吵架”的行为动作可能已经发生, 也可能还未发生，说话人用“何必”反问以表达他反对的态 度。整个句子隐含的语义是: 既然一个老头住哪都没差别, 就不要为这事而吵架。再如:

（34）桂英如此想着, 想到将要走的人了, 何必临走 还落个恶名, 她索性忍耐。

这个句子的前提是“桂英选择忍耐”，“临走还落个恶 名”是表达未然的事, 说话人用“何必”其义显然是表达否 定。整个句子隐含的语义是: 既然选择忍耐, 就不要临走 时落个恶名。

表2“何必”的语义条件。

\begin{tabular}{ll}
\hline 结构 & 例句 \\
\hline 何必 + 前提 & 何必一定要我当着大家的面说 \\
何必 + 动作 + 前提 & 何必一口答应下来, 我们有房有车, 也不差这 \\
前提 + 何必 + 动作 & 你貌美心善, 何必找一个歪瓜裂疋的男友 \\
\hline
\end{tabular}

\subsection{2. 何苦}

上已有述，“何必”与“何苦”最大的不同在于其表达的 语气以及出现的语义条件不同。那么, “何苦”表达的语气 和出现的语义条件又是什么呢? 也请看下面例子:

（35）为什么? 你何苦做这种憋屈的事情呢?

这话虽短, 但它其实已含有 “说话人认为这种事不值 得做”的完整语义, 因为“憋屈”一词已是句义表达隐含的 前提条件了。

（36）王琦瑶不同意, 她反正是逃不了了, 何苦再赔 上一个。

从这个例子可以看出, 句的前提是“王琦瑶逃不掉”, “再赔上一个人”也许是已完成或还未完成的行为动作, 说 话人使用“何苦”反问以表达他的态度。因此, 其语义是: 既然知道王琦瑶逃不掉, 那再赔上一个人就不值得了。

（37）“你何苦收藏那些书?何苦反封建? 你害自己下 地狱还不够, 还害了咱女儿, 还把我害了...”

句子的前提是“不仅害了他自己, 还害了女儿, 害了 我”，说话人以“何苦”反对他“收集反封建的书”。

表3“何苦”的语义条件。

\begin{tabular}{ll}
\hline 结构 & 例句 \\
\hline 何苦+前提 & 你何苦抓着这种小事不放 \\
何苦 + 动作 + 前提 & 何苦这样累死累活的供他读书, 反正他都是个 \\
前提 + 何苦+动作 & 早不起的阿辻终究要别离, 他何苦还用心良苦地挽留 \\
\hline
\end{tabular}

\section{4. 语气副词“何必/何苦”在对外汉语教学中的运 用}

\section{1. 对汉语学习者的偏误分析}

所谓偏误, 是由于没有掌握好目标语所产生的一种规 律性错误, 它偏离了目标语, 这是缺乏语言能力所造成的。 这里通过HSK动态作文语料库的材料, 分析汉语学习者学 习“何必”与“何苦”时所出现的偏误现象。这些偏误现象大 体上可以归纳为遗漏、错序、误代几种类型。

\section{1. 遗漏}

第一个研究对外汉语教学中留学生偏误问题的学者 应该是鲁健骥。在《外国人学汉语的语法偏误分析》一文 中, 他根据偏误性质将其分为遗漏、误加、误代及错序四 个大类。[14]遗漏是指词语或句子中遗漏了某个或某几个 成分所导致的一种偏误现象。例如:

（38）何必（）我干活呢？（日本女）

这个例子所产生的偏误是汉语学习者遗漏了反诘语 气副词后的“让”之类的使令动词, 使得句子既不文从义顺, 也无法表达出有人要强令自己干活而 “我”认为没必要这 一使令语义。遗漏“让”的原因, 其一可能还未完全掌握它 的语义和用法, 其意义较为“抽象”; 其二也许说话时其关 注的重点在于 “干活”上, 忽略了究竟是自愿干活还是有 人要“我”干这一关键点上, 致使语句失去了使令意义, 因 而无法准确地表达语句原本要传达的语义。

（39）既然我都决定去中国学习了，何苦（）？（美 国 男) 
在这个偏误句中, “何苦”之后遗漏了必要的成分, 致 使句子残缺不全。上已有述，“何苦”的语义条件是以“前 提＋何苦＋动作”为结构的。而本句“何苦”之后遗漏了表 示动作的成分，使其无法表达完整的句义。造成这一原因 可能有两种情况: 一是记忆出现了偏差, 二是母语的负迁 移。在英语中, why是可以表示反诘语气的, 在极度无奈 的情况下有时可以省略后面的动词成分, 直接用why表示 无可奈何。比如, 夫妻吵架时, 女方不依不饶, 男方则会 抓狂地说“I have apologized to you,why?”why后省略了 “You're still angry”。所以, 以英语为母语的人常常遗漏“何 苦”之后的动词成分, 很有可能是母语负迁移所引起的。 [15]

针对遗漏这种偏误, 教学时可以从先从整体入手。对 于初学者来说, 要想全面理解、掌握“何必”“何苦”的语义、 语法、语用方面是很难的。如果初学者死记硬背其外部结 构, 没有深入到内部, 不仅难知其然, 更不知其所以然, 就会出现该用则未用的遗漏现象。因此, 教学时要从整体 出发, 先理清两个词的共同点与用法, 在有了基础以后, 重点要进行口语交际训练, 让其对反诘语气有深入的理解。 有了整体把握后, 就可以对初学者进行细致讲解, 包括其 分布特征, 可与什么词性的词语搭配, 其语义特征和语义 条件是什么, 等等。与此同时, 除加强学生的口语交际能 力外, 还要强化他们的阅读能力, 让他们在不断变化的语 言环境中得到正确地运用, 并能准确地表达语义。相信经 过多个回合系统强化训练, 完全能够掌握其语义用法。

2. 错序

错序这种偏误现象是由于句中某个或几个成分所放 位置顺序有误而导致的。汉语是无形态变化的词根语, 主 要依靠虚词和语序来表示语义的。所以, 语序在汉语中具 有显著地位, 而要掌握句中不同成分所处的不同语序, 这 对于汉语初学者来说也成了一大难题。例如:

（40）如果可以用手机买东西，（）用现金何必呢? (韩国 女)

（41）和老师何苦争执, 老师比我知道得多。（泰国 女)

上文分析了“何苦”“何必”的语义条件，例41的语序应 为: “如果可以用手机买东西, 何必用现金呢? ”这符合“前 提十何必十动作”语序结构模式, “何必”之后没有出现“买” 这一动词, 这是承前省略。根据“何苦十事件十前提”这一 语序模式, 例 42 的语序应该是：“何苦和老师争执，老师 比我知道得多。”而原句错误地将“何苦”置于事件之后, 以致造成句子的偏误。

产生这种偏误的原因, 可能是目标语的过于泛化。教 学时, 教师讲反诘语气副词通常要强调副词共有的语法特 性, 这样学生就认为副词一般修饰动词或形容词, 用作状 语。这样一来, 他们就将反诘语气副词与一般副词等同起 来, 然后根据语法规则, 将副词置于谓语动词前, 这就可 以解释为什么学生会将“何苦”放在动词“争执”前了。如果 忽视“何必”“何苦”的反诘语气特性, 仅视之一般副词, 势 必会导致句子的错序。

如要减少或杜绝错序这一偏误现象, 教师既要讲清 “何苦”“何必”作为副词类别所具有的一般共性, 也要讲透
二者作为反诘语气副词所具有的特殊个性。[16]惟有这样, 才能让学生在实际交际中得心应手, 运用自如。

3. 误代

误代偏误是由于从两个或几个形式中选取了不适合 于特定语言环境的那一个从而造成的偏误。这两个或几个 形式,或者是意义相同或相近,但用法不同;或者只是形式 上有某种共同之处(如字同), 而意义和用法不同;或者是用 法相同,意义相反。例如:

（42）因为不让他安乐死, 他早晚也得死, 那何等呢 (何苦) ? (新加坡 女)

（43）为了一个人的反对, 怀疑自己的能力, 何不呢 （何必）?（韩国 女）

词语的误代可以是几个意义相同或相近, 而用法不同; 也可能是形式相似 (如字同形近) 而意义完全不同; 或者 用法相同而意义相反[11]。这几种情况, 很大程度上是学 习者对词义语用理解不透所导致的。[17]

例 42 属于第二种情况, 形近义不同。“何等”指“什么 样”的, 表示疑问; 也有“多么”的意思, 用于表示“不寻常” 的感叹语气。例如: “你猜她是何等女子? ”“国家大事, 何等重大。”一个用于疑问句表示疑问, 一个用于陈述句 表感叹。很显然, 它与“何苦”的意义完全不同。例43则属 于第三种情况，其用法相同而意义则相反。“何不”是“应 该”或“可以”义，与“何必”所表示的“不必”完全相反，但是 二者都可在句末加语气词“呢”。例如: “如果买满100元就 可以减三十元, 何不呢? ”可以看出, 学习者没有理解其 词义, 所以混淆不清, 以致出现了误用。

误代虽产生于学生对词义的理解问题, 但究其根本, 也与教师和教材有很大关系。在汉语母语者看来, “何不” 与“何必”“何苦”与“何等”其义有天壤之别, 这也让很多人 想当然地认为汉语学习者只要通过阅读词语解释就可以 将二者轻易地区分开来。但是, 事实并非如此。如果仅 靠阅读课本词语解释, 学生是无法透彻理解, 也难以将 其区分开来的。众所周知, 汉语初学者的课本中的汉语 生词是以英语作注解的, 而英语与汉语差异很大的, 二 者的意义并非一一对等的, 很多时候很难用英语准确地 表达词语间的细微差异。如“何苦”与“何必”都可译成 “why bother to”, 教材没有根据两个词的句法分布、语义 条件以及语用预设等方面去作详细的解释, 学生也就一 头雾水。所以, 教材应该与时俱进, 不仅要细致辨析同 义词、近义词之间的细微差异, 还要根据语境列举大量 例句供学生去体会, 去模仿, 去运用。只有这样, 才能 提高学生的语言运用能力。

\section{2. 对汉语学习者的教学策略}

汉语中的词是音、形、义统一体, 汉语母语者是先学 字音, 再学字形, 最后理解字义的。学词, 是将字义组合 起来, 并以类推的方法来理解词义。但是, 在对外汉语教 学中, 教师则把语法教学作为它的重点, 这在某种程度上 忽略了字与词之间的联系, 这使学生无法把握汉语词汇的 规律, 致使他们无法了解不同的语境, 每个词的搭配对象 和语义特征是不同的, 所以汉语初学者掌握汉语词汇遇到 了很大的困难。 
不过, “语素教学法”的出现, 对于把握汉语的本质有 重要意义。这是因为它抓住了语言中的最小单位一一语素 来进行教学, 这一理论一定程度上能让学习者更好地掌握 汉语词汇。在此就借助语素教学法以及语境教学法将“何 苦”与“何必”拆分，从“何”“必”“苦”三个语素内涵出发，从 整体一一个体一整体进行讲授, 再设置适当的情景语境, 以帮助学生更好地掌握这对反诘语气副词。

教学对象: 中级汉语学习者

教学步骤:

1. 朗读对话

对话 1:

张亮: “小李, 午饭做好了, 我们一起吃饭吧。”

李梅: “不用了, 我想去外面吃饭。”

张亮: “我已经做好了午饭, 你何必去外面吃呢? ”

对话2:

王杰: “今天天气不好, 你最好不要出门。”

李丽: “我想要去看电影。”

王杰: “外面下着雨, 你何苦冒雨出门呢? ”

2. 以语素教学法阐释“何必何苦”的语义用法

先从整体出发, 说明“何必”与“何苦”都是用来表示反 问语气的语气副词, 明确两个词最重要的语法功能, 给学 生留下第一印象。再分解词语, 将这两个词拆分为 “何”“必”“苦”, 最后再将它们组合成词进行形象的解释, 以加深学生的印象。

先跟学生解释“何”的意义, “何”由疑问代词逐渐变为 表反问语气副词, 是有历史来源的。但是, 对于古代汉语 不了解或了解甚少的汉语学习者来说, 没有必要详细阐释 “何”的波源关系。所以, 我们只要求学生记住“何”在反诘 语气副词这一整体中的最大语用特点——表示反问语气 就可以了。这样, 先让他们在脑海中固定“何”的功能和意 义，这有利于对“苦”和“必”的理解。

教学语素“必”, 应依据它在汉语中的本义进行扩展性 的讲释。可以告诉学生“必”古时是指武器的把手, 一个军 人想要在战场上活下来且获得胜利, 他的兵器质量一定要 好。否则, 兵器的把手折断了, 那么就失去生命和胜利的 保证。所以, “必”有“一定、必须”的意思。而语素“何、必” 组合在一起, 表达“为什么一定要”的意思。[18]

而语素“苦”, 则可从其所具的形象感入手。“苦”最初 指一种味道极其苦澀的植物, 从这引申出“辛苦”“痛苦”。 [19]所以, 我们可以告诉学生, “苦”就像吃了balsam pears (苦瓜) 一般的滋味, 让人觉得痛苦。而“何、苦”这两个 语素组合, 可以形象地理解成“为什么要让自己像吃了苦 瓜一样感到痛苦呢”, 即“不要做让自己感到痛苦的事情”。

3. 以句法规则构拟“何必何苦”的结构模式

(1)何必:

分句 1 , 主语 + 何必 + 动词或动词性短语 + 呢?

分句 1 表示的是句子的前提条件, 即说话人认为这件 事没必要做的原因; 动词或动词性短语则是受话人执意要 进行的行为动作或事件, 这就是说话人劝说受话人的关键 所在, 其后的语气词可以省略。

(2)何苦:

分句 1 , 主语 + 何苦 + 动词或动词性短语 + 呢?
分句 1 重点在于说话人认为受话人做这个行为动作会 让受话人自己感到痛苦, 所以劝阻对方不要做让自己难以 忍受的事情。

(3)“何必/何苦”独立成句何必/何苦十呢！

“何必/何苦”两个副词, 一般情况下能加上语气词“呢” 独立成句。

\section{4. 设置“何必何苦”运用的特定语境}

先设置一个情景, 让学生根据语境运用所学的词语造 句和对话。接着, 又让他们独立设置语境进行交际活动。 教师可以根据语境的设置以及句子运用的正确与否来判 断他们的学习效果。

情景 1: 今天没有作文课, 但是John的书包里却带了 作文书, Lucy发现后告诉了John, 她认为做这件事没有必 要, 所以劝他不要带。

例句: “今天没有作文课，你何必带作文书呢? ”

情景 2: rose想去剪头发, 但是Mike认为短发不适合 她, 剪短发可能会让rose没有之前那样漂亮, 所以他希望 rose不要去剪发, 不要做那种让自己感到痛苦的事情。 呢?"

例句: “你剪短发可能没有现在好看，你何苦去剪发

很有可能学生是第一次接触“何必/何苦”这类反诘语 气副词, 教师必须设计出适合汉语学习者的教学方案, 讲 透其意义和用法, 为学生以后学习反诘语气副词打下扎实 的基础。

\section{5. 结语}

综上所述, 本文分析了反诘语气副词“何必/何苦”语义、 语法、语用功能及其在对外汉语教学中的应用问题, 发现 二者在语义、语法、语用等方面存在一定的差异, 与此同 时还对汉语初学者所出现的偏误现象进行了分析, 并就这 一问题制定了有效的教学方案。

通过分析发现，从其语法位置看，“何必/何苦”大多位 于句中, 少数位于句首或句末; 从其词语搭配看, “何必/ 何苦”可以与不同行为动词搭配, 还可以带语气词“呢”, 受频率副词“又”等修饰; 从其语义轻重看, “何必/何苦” 语义表达有轻重程度之分, 后者比前者的语义要重; 从其 语用功能看, “何必”的语用预设一般是行为动作已经完成 或尚未完成, 但是说话者认为这一行为动作没有必要实施, 而受话者则认为这一行为动作可以或应该实施, 在他看来 是合理的。“何苦”的语用预设是: 行为动作还未发生或已 经发生, 受话者有理由实施这一行为动作, 而说话者主观 认为做这件事是没有必要的。

大多数情况下，“何苦”是可以替换“何必”的。但是, 如以做某事会带来某种消极后果时, 一般用“何苦”而不能 不能用“何必”。“何必”与“何苦”虽然都带有主观性和规劝 性的语义特征, 但“何必”具有强调、建议的意味, 而“何 苦”则偏重于惋惜、无奈的色彩。

本文根据 HSK动态语料库对汉语学习者学习 “何 必”“何苦”时所犯的错误进行了分析, 着重地分析了遗漏、 错序和误代三种偏误类型, 并据此运用语素教学法为对外 汉语教学设计了一套教学方案, 力图为施教者提供有益的 借鉴或启发。 


\section{参考文献}

[1] 王力. 中国现代语法[M].北京：商务印书馆,2011。

[2] 史金生.语气副词的范围、类别的共现顺序 [J]. 中国语 文,2003(01)。

[3] 肖奚强.非典型模态副词句法语义分析 [J]. 语言研 究,2003(04)。

[4] 何雪飞.早期现代汉语的副词使用情况考察[D].重庆师范大 学学位论文.2019。

[5] 范宁.“何”的词汇化研究[D]. 黑龙江大学学位论文, 2010。

[6] 张晓东.对外汉语教学中语气副词“倒”的探究 [J].长春教育 学院学报.2019(09)。

[7] 武远佳.语气副词“早晚”的形成时代及其过程[J].浙江万里 学院报.2019(13)。

[8] 袁静.语气副词“不必”和“无须”的共时比较 [J].湖北科技学 院学报.2018(12)。

[9] 朱宇贺.语气副词“想来”和“看来”的比较分析 [J].吉林省教 育学院学报.2019(39)。

[10] 张谊生. 现代汉语副词研究 $[\mathrm{M}]$.上海: 学林出版社,2001。

[11] 林华勇.现代汉语副词研究回顾[J].汉语学习, 2003(01)。

[12] 朱晓军,郭静婷.“何”类反诘语气副词对比浅析一以以“何必” 与“何苦”、“何尝”与“何曾”的对比为例 $[\mathrm{J}]$. 河南师范大学学 报(哲社科版),2014(06)。

[13] 鲁健䩀.外国人学汉语的语法偏误分析 [J]. 语言教学与研究, 1994(01)。

[14] 胡孝斌.反问句的话语制约因素[J].世界汉语教学,1997(01)。

[15] 郭继禁.反问句的语义语用特点 [J].中国语文.1997(02)。

[16] 赵雷.谈反问句教学 [J]. 语言教学与研究, 2003(03)。

[17] 楚艳芳. “何”的用法及其语法化过程 [J]. 德州学院学 报,2009(02)。

[18] 李宇明.程度与否定 [J]. 世界汉语教学, 1999(01).

[19] 沈家煊.语言的“主观性”和“主观化”[J]. 外语教学和研 究,2001(04)。 